\title{
THE RIEMANN PROBLEM FOR \\ NONCONVEX SCALAR CONSERVATION LAWS AND HAMILTON-JACOBI EQUATIONS
}

\begin{abstract}
STANLEY OSHER
ABSTRACT. We present a closed form expression for the viscosity solution to the Riemann problem for any scalar nonconvex conservation law. We then define an analogous problem for any scalar nonconvex Hamilton-Jacobi equation and obtain its (even simpler) solution. Extensions to two (and by inference, higher) space dimensional problems, when the initial discontinuity lies on a hyperplane, are also given.
\end{abstract}

We shall be concerned with solutions to the following nonlinear scalar partial differential equations:

$$
\varphi_{t}+f\left(\varphi_{x}\right)+g\left(\varphi_{y}\right)=0
$$

and

$$
u_{t}+f(u)_{x}+g(u)_{y}=0
$$

for $t>0$, with special initial data.

This restriction to two space dimensions is made for simplicity only.

A rather complete existence and uniqueness theory for the Cauchy problem for (CL) has been known for some time (see, e.g. [2]). Recently a parallel theory was developed for $(\mathrm{H}-\mathrm{J})$ [1]. (More general equations have also been treated by both of these theories.)

Solutions of (CL) are well known to generally develop discontinuities, even if the initial data are smooth. Solutions of $(\mathrm{H}-\mathrm{J})$ stay continuous, but generally develop jumps in derivatives. To obtain uniqueness and the rest of the theory mentioned above, it suffices to consider only viscosity solutions.

A solution to (1) is a viscosity solution if it is the local uniform limit of $\varphi^{\varepsilon}(x, y, t)$ as $\varepsilon \downarrow 0$, where $\varphi^{\varepsilon}$ solves

$$
\varphi_{t}^{\varepsilon}+f\left(\varphi_{x}^{\varepsilon}\right)+g\left(\varphi_{y}^{\varepsilon}\right)=\varepsilon\left(\varphi_{x x}^{\varepsilon}+\varphi_{y y}^{\varepsilon}\right)+G_{\varepsilon}\left(x, y, t, \varphi^{\varepsilon}, \varphi_{t}^{\varepsilon}, \varphi_{x}^{\varepsilon}, \varphi_{y}^{\varepsilon}\right) .
$$

Here, $G_{\varepsilon}$ converges uniformly to zero on compact subsets of $R^{2} \times R^{+} \times R^{4}$.

Received by the editors March 21, 1983.

1980 Mathematics Subject Classification. Primary 35L60, 35L65, 35L99.

Key words and phrases. Conservation law, Hamilton-Jacobi equations, Riemann problem.

'Research supported by NSF Grant MCS 82-007788, NASA University Consortium Agreement NCA2-0R390-202, and ARO Grant DAAG29-82-K0090. 
A viscosity solution to (2) is the local $L^{1}$ limit of $u^{\varepsilon}(x, y, t)$, as $\varepsilon \downarrow 0$, where $u^{\varepsilon}$ solves

$$
u_{t}^{\varepsilon}+f\left(u^{\varepsilon}\right)_{x}+g\left(u^{\varepsilon}\right)_{y}=\varepsilon\left(u_{x x}^{\varepsilon}+u_{y y}^{\varepsilon}\right) .
$$

All solutions obtained below will be viscosity solutions.

The Riemann problem for $(\mathrm{CL})$ in one space dimension is the following. Solve

$$
u_{t}+f(u)_{x}=0
$$

for $t>0$, with initial data

$$
u(x, 0) \equiv u^{L}, \quad x \leqslant 0 \quad \text { and } \quad u(x, 0) \equiv u^{R}, \quad x>0,
$$

for given constants $u^{L}, u^{R}$.

We have recently [4] obtained a simple formula for the solution to this problem for general $f$. For completeness, we shall present the formula here, and outline its derivation below.

The solution to (3) is well known to have the following properties (see e.g. [3]):

(i) $u(x, t)=u(x / t)=u(\zeta)$,

(ii) $u(\zeta)$ lies between $u^{L}$ and $u^{R}$,

(iii) $u(\zeta) \equiv u^{L}$, if $\zeta<-N, u(\zeta) \equiv u^{R}$, if $\zeta>N$, for some $N>0$.

We have

LEMMA 1. If $u^{l}<u^{R}$, then

$$
\zeta u(\zeta)-f(u(\zeta))=\max _{v \in\left[u^{I}, u^{R}\right]}[\zeta v-f(v)]
$$

or if $u^{L}>u^{R}$, then

$$
\zeta u(\zeta)-f(u(\zeta))=\min _{v \in\left[u^{R} \cdot u^{l}\right]}[\zeta v-f(v)]
$$

It is easy to see that the above Legendre transforms are uniformly Lipschitz continuous, with Lipschitz constant $\max \left[\left|u^{L}\right|,\left|u^{R}\right|\right]$, and are convex and concave, respectively, as functions of $\zeta$. Taking the distribution derivative of equations (4) gives us an expression for $u(\zeta)$ :

THEOREM 1. If $u^{L}<u^{R}$, then

$$
u(\zeta)=\frac{d}{d \zeta}\left(\max _{v \in\left[u^{L}, u^{R}\right]}[\zeta v-f(v)]\right) .
$$

If $u^{L}>u^{R}$, then

$$
u(\zeta)=\frac{d}{d \zeta}\left(\min _{v \in\left[u^{R}, u^{L}\right]}[\zeta v-f(v)]\right) .
$$

The analogous problem for $(\mathrm{H}-\mathrm{J})$ in one space dimension is

$$
\varphi_{t}+f\left(\varphi_{x}\right)=0
$$

for $t>0$, with initial data

$$
\varphi(x, 0) \equiv x u^{L}, \quad x \leqslant 0 \quad \text { and } \quad \varphi(x, 0) \equiv x u^{R}, \quad x>0,
$$

and given constants $u^{L}, u^{R}$. 
We have

THEOREM 2.

$$
\begin{aligned}
\varphi(x, t) & =t[\zeta u(\zeta)-f(u(\zeta))] \\
& =t\left(\max _{v \in\left[u^{L}, u^{R}\right]}[\zeta v-f(v)]\right), \quad \text { if } u^{L}<u^{R} \\
& =t\left(\min _{v \in\left[u^{R}, u^{L}\right]}[\zeta v-f(v)]\right), \quad \text { if } u^{L}>u^{R}
\end{aligned}
$$

This last formula points out an interesting link between the Riemann problems for (CL) and (H-J).

In two space dimensions, we restrict ourselves to the following problems:

$$
u_{t}+f(u)_{x}+g(u)_{y}=0
$$

for $t>0$, with initial data

$$
\begin{gathered}
u(x, y, 0) \equiv u^{L}, \quad \text { if } x \sin \theta-y \cos \theta \leqslant 0 \\
u(x, y, 0) \equiv u^{R}, \quad \text { if } x \sin \theta-y \cos \theta>0 \\
\varphi_{t}+f\left(\varphi_{x}\right)+g\left(\varphi_{y}\right)=0
\end{gathered}
$$

for $t>0$, with initial data

$\varphi(x, y, 0) \equiv(x \sin \theta-y \cos \theta) u^{L}+(x \cos \theta+y \sin \theta) u^{M}, \quad$ if $x \sin \theta-y \cos \theta \leqslant 0$. $\varphi(x, y, 0) \equiv(x \sin \theta-y \cos \theta) u^{R}+(x \cos \theta+y \sin \theta) u^{M}, \quad$ if $x \sin \theta-y \cos \theta>0$.

Here $u^{L}, u^{M}, u^{R}$ and $\theta$ are fixed constants, with $0 \leqslant \theta<\pi$.

For (CL), we gave pieceiwse constant initial data, with a single jump across a plane through the origin. For $(\mathrm{H}-\mathrm{J})$, we prescribed $\left(\varphi_{x}, \varphi_{y}\right)$ to be constant, except for a single jump in normal derivative across a plane through the origin. We also require that $\varphi$ be continuous, and, for convenience only, set $\varphi(0,0,0)=0$.

We define

$$
\zeta=\frac{x \sin \theta-y \cos \theta}{t}
$$

and state

THEOREM 3. The solution to (8) is

(10a) $u(\zeta)=\frac{d}{d \zeta}\left(\max _{v \in\left[u^{L}, u^{R}\right]}[\zeta v-(f(v) \sin \theta-g(v) \cos \theta)]\right), \quad$ if $u^{L}<u^{R}$,

or

(10b) $u(\zeta)=\frac{d}{d \zeta}\left(\min _{v \in\left[u^{R}, u^{L}\right]}[\zeta v-(f(v) \sin \theta-g(v) \cos \theta)]\right), \quad$ if $u^{L}>u^{R}$ 
THEOREM 4. The solution to (9) is

$$
\begin{aligned}
\varphi(x, y, t)= & u^{M}(x \cos \theta+y \sin \theta) \\
& +t\left(\operatorname { m a x } _ { v \in [ u ^ { I } , u ^ { R } ] } \left(\zeta v-f\left(v \sin \theta+u^{M} \cos \theta\right)\right.\right. \\
& \left.\left.-g\left(-v \cos \theta+u^{M} \sin \theta\right)\right)\right), \quad \text { if } u^{L}<u^{R},
\end{aligned}
$$

or

(11b) $\varphi(x, y, t)=u^{M}(x \cos \theta+y \sin \theta)$

$$
\begin{aligned}
+t\left(\operatorname { m i n } _ { v \in [ u ^ { R } , u ^ { l } ] } \left(\zeta v-f\left(v \sin \theta+u^{M} \cos \theta\right)\right.\right. \\
\left.\left.-g\left(-v \cos \theta+u^{M} \sin \theta\right)\right)\right), \quad \text { if } u^{L}>u^{R} .
\end{aligned}
$$

Proofs. We begin by outlining the proof of Lemma 1 , for $\zeta=0$. It is known that the viscosity solution of (3) is a nondecreasing function of its initial data. In [4], we showed that this fact implies that the function $h\left(u^{R}, u^{l}\right)=f(u(0))$ is a nonincreasing function of its first argument, and nondecreasing function of its second.

For any $u$ between $u^{l}$ and $u^{R}$, we consider

$$
\begin{aligned}
\operatorname{sgn}\left(u^{R}-u^{\prime}\right)\left[h\left(u^{R}, u^{\prime}\right)-f(u)\right]= & -\operatorname{sgn}\left(u^{\prime}-u\right)\left[h\left(u^{R}, u^{\prime}\right)-h\left(u^{R}, u\right)\right] \\
& +\operatorname{sgn}\left(u^{R}-u\right)\left[h\left(u^{R}, u\right)-h(u, u)\right]=\mathrm{I}+\mathrm{II} .
\end{aligned}
$$

By our previous remarks, both I and II are nonpositive, hence

$$
\operatorname{sgn}\left(u^{R}-u^{L}\right)[f(u(0))-f(u)] \leqslant 0,
$$

for all $u$ between $u^{R}$ and $u^{L}$. Since $u(0)$ lies in this range, we have Lemma 1 , for $\zeta=0$.

To obtain Lemma 1 for all $\zeta$, we make a change of independent variables

$$
y=x-s t, \quad \tau=t .
$$

The resulting problem is still (3), with $f(u)$ replaced by $f(u)-s u$. The previous argument gives us Lemma 1 , for $\zeta=s$.

Theorem 1 follows from taking the distribution derivative of (4) (see [4]).

To verify Theorem (2), we first compute

$$
\varphi_{t}=\zeta u(\zeta)-f(u(\zeta))-t u(\zeta) \zeta_{t}=-f(u(\zeta)), \quad \varphi_{x}=t u(\zeta) \zeta_{x}=u(\zeta)
$$

Thus, equation (6) is satisfied.

Letting $t \downarrow 0$ shows us that the initial data is taken on. 
It only remains to show that $\varphi$ is a viscosity solution. Since $\varphi_{x}=u(\zeta)$, we have, for $\bar{x}<-N t$

$$
\begin{aligned}
\varphi(x, t) & =\bar{x} u^{L}-t f\left(u^{L}\right)+\int_{\bar{x}}^{x} u\left(\frac{s}{t}\right) d s \\
& =\bar{x} u^{L}-t f\left(u^{L}\right)+\lim _{\varepsilon \downarrow 0} \int_{\bar{x}}^{x} u^{\varepsilon}(s, t) d s \\
& =\lim _{\varepsilon \downarrow 0}\left[\bar{x} u^{L}-t f\left(u^{L}\right)+\int_{\bar{x}}^{x} u^{\varepsilon}(s, t) d s\right] \\
& =\lim _{\varepsilon \downarrow 0} \varphi^{\varepsilon}(s, t) .
\end{aligned}
$$

Here

$$
\varphi_{t}^{\varepsilon}+f\left(\varphi_{x}^{\varepsilon}\right)-\varepsilon \varphi_{x x}^{\varepsilon}=f\left(u^{\varepsilon}(\bar{x}, t)\right)-f\left(u^{L}\right)-\varepsilon u_{x}^{\varepsilon}(\bar{x}, t) .
$$

It is easy to see that the function of $t$, defined on the right above, converges uniformly to zero on compact subsets of $0<t<\bar{x} / N$, as $\varepsilon \downarrow 0$. Thus $\varphi$ is a viscosity solution.

To prove Theorem 3, we make an orthogonal change of variables

$$
\left(\begin{array}{c}
z \\
w
\end{array}\right)=\left(\begin{array}{cc}
\sin \theta & -\cos \theta \\
\cos \theta & \sin \theta
\end{array}\right)\left(\begin{array}{l}
x \\
y
\end{array}\right) \Leftrightarrow\left(\begin{array}{l}
x \\
y
\end{array}\right)=\left(\begin{array}{cc}
\sin \theta & \cos \theta \\
-\cos \theta & \sin \theta
\end{array}\right)\left(\begin{array}{c}
z \\
w
\end{array}\right) .
$$

The solution $u^{\varepsilon}$, to the regularized problem solves

$$
u_{t}^{\varepsilon}+\left(\sin \theta f\left(u^{\varepsilon}\right)-\cos \theta g\left(u^{\varepsilon}\right)\right)_{z}+\left(\cos \theta f\left(u^{\varepsilon}\right)+\sin \theta g\left(u^{\varepsilon}\right)\right)_{w}=\varepsilon\left(u_{z z}^{\varepsilon}+u_{w w}^{\varepsilon}\right)
$$

for $t>0$, with initial data

$$
u^{\varepsilon}(z, w, 0) \equiv u^{L}, \quad \text { if } z \leqslant 0 \quad \text { and } \quad u^{\varepsilon}(z, w, 0) \equiv u^{R}, \quad \text { if } z>0 .
$$

It is clear that the solution will be independent of $w$, and hence, by virtue of Theorem 1, will converge as $\varepsilon \downarrow 0$, to (10).

For the proof of Theorem 4 , we note that the same change of variables gives us

$$
\begin{gathered}
\varphi_{t}+f\left(\varphi_{z} \sin \theta+\varphi_{w} \cos \theta\right)+g\left(\varphi_{z}(-\cos \theta)+\varphi_{w} \sin \theta\right)=0, \\
\varphi(z, w, 0)=u^{L} z+u^{M} w, \quad \text { if } z \leqslant 0 \text { and } \varphi(z, w, 0)=u^{R} z+u^{M} w, \quad \text { if } z>0 .
\end{gathered}
$$

Let $\varphi=\psi+u^{M} w$. Then $\psi$ satisfies

$$
\begin{gathered}
\psi_{t}+f\left(\psi_{z} \sin \theta+u^{M} \cos \theta\right)+g\left(\psi_{z}(-\cos \theta)+u^{M} \sin \theta\right)=0 \\
\psi(z, w, 0) \equiv u^{L} z, \quad \text { if } z \leqslant 0 \quad \text { and } \quad \psi(z, w, 0) \equiv u^{R} z, \quad \text { if } z>0 .
\end{gathered}
$$

Then, since $\psi$ is obviously independent of $w$, we may use Theorem 2 to obtain the viscosity solution for this one-dimensional problem. This leads us to (11). It is easy to show that the resulting function, $\varphi$, is a viscosity solution for (9). We merely use a simple modification of the argument used in the proof of Theorem 2.

Acknowledgement. We would like to thank Professor W. G. Strang for several helpful discussions. 


\section{BIBLIOGRAPHY}

I. M. G. Crandall and P. L. Lions, Viscosity solutions of Hamilton-Jacobi equations, Trans. Amer. Math. Soc. 277 (1983), 1-42.

2. S. N. Kružkov, First order quasi-linear equations in several independent variables, Math. USSR Sb. 10 (1970), 217-243.

3. P. D. Lax, Hyperbolic systems of conservation laws and the mathematical theory of shock waves, CBMS Regional Conference Ser. in Appl. Math., no. 11, SIAM, Philadelphia, Pa., 1972.

4. S. Osher, Riemann solvers, the entropy condition, and difference approximations, SIAM J. Numer. Anal. (to appear).

Department of Mathematics, University of California, los Angeles, California 90024 